\section{COVID-19: The possible medical strategies}

\section{Mohamed SA Mohamed*}

MSAM for Medical Consultations, Thoracic Transplantation Department, University Clinic Essen, Essen, Germany

\section{Abstract}

In late 2019, a pandemic crisis started in Wuhan, China, swept the whole world. The disease is caused by the SARS-CoV- 19 virus that belongs to the corona family of viruses. The virus mainly caused failure of respiration, and led to many deaths worldwide. The main focus of research and medicine is to find more about the virus, as well as the development of effective preventive and therapeutic measures. While many trials and opinions have been published, which might support or contradict each other, this article tries to provide a simplified viewpoint about the disease. We highly recommend the therapeutic strategies to include drug combinations that can target the pathogenesis at many levels. For example, a combination of an effective anti-viral Remdesivir, soulable ACE2, and an immune modulator.

COVID-19 is a pandemic caused by SARS-CoV-2 virus, which targets the Pneumocytes II in the lungs. The virus enters the target cells by attaching to ACE2 surface receptors. SARS-CoV-2 virus is + ssRNA, which means;

- It replicates its genetic material through RNAdependent RNA polymerase

- It translates its proteins in cell ribosomes

- Newly synthesized RNA and proteins assemble and spread to new cells as the infected cells die [1].

What happens in the lungs?

The infection stimulates the resident macrophages and immune cells to start a call of inflammation. This involves the secretion of important mediators; IL1 $\beta$, IL6 \& TNF $\alpha$, and increased vascular permeability. This leads to the following:

- Infected cells in the lungs die

- The surfactant production decreases

- Alveoli collapse

- Alveolar odema develops

- The call of inflammation may result in massive production of cytokines, the so called cytokine storm, which leads to Multi-Organ Failure

\section{Diagnosis} AJR.20.22976

\section{More Information}

*Address for Correspondence: Mohamed SA Mohamed, MSAM for medical consultations,

Thoracic Transplantation Department, University Clinic Essen, Essen, Germany,

Email: mohammed.shehatta1@gmail.com

Submitted: 24 April 2020

Approved: 08 June 2020

Published: 09 June 2020

How to cite this article: Mohamed MSA. COVID-19: The possible medical strategies. Int J Clin Virol. 2020; 4: 071-075

DOI: 10.29328/journal.ijcv.1001018

Copyright: @ 2020 Mohamed MSA. This is an open access article distributed under the Creative Commons Attribution License, which permits unrestricted use, distribution, and reproduction in any medium, provided the original work is properly cited.

Keywords: COVID-19; SARS-CoV-2;

Respiratory failure; Multi-organ failure

A) Check for updates

OPEN ACCESS

Clinical symptoms and signs may include fever, dry cough, sore throat and shortness of breath. The findings of CT thorax can show the progression of viral pneumonia (Figure 1).
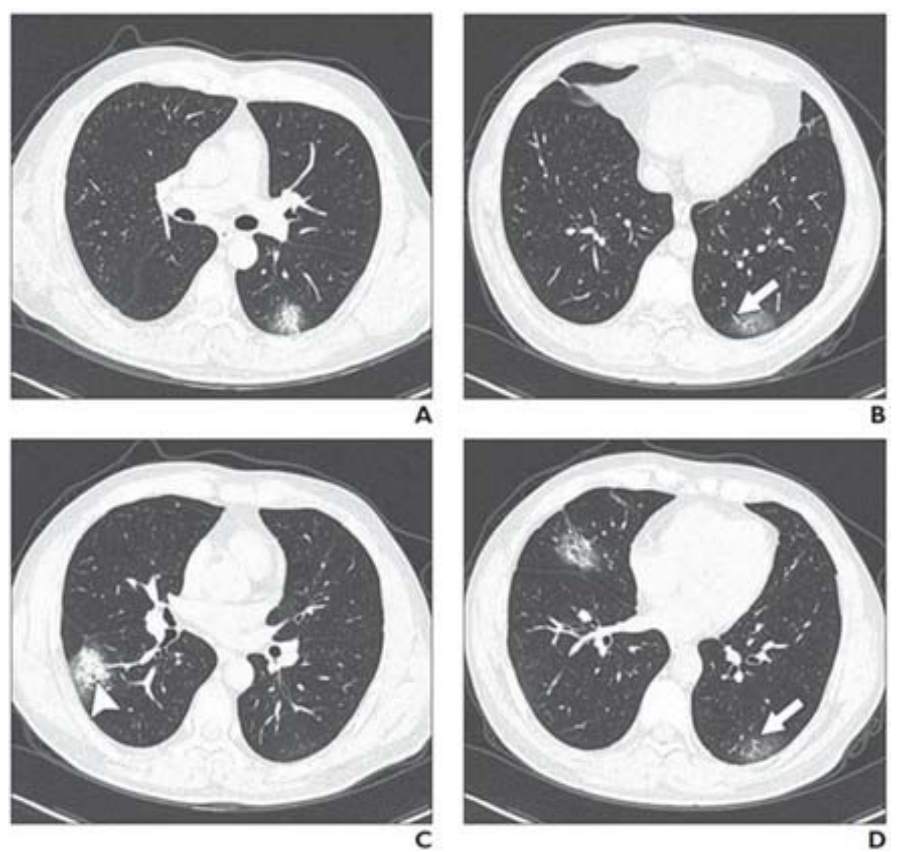

Figure 1: Some possible CT findings in Covid-19 lung infection.

Source: Wei Zhao, et al. American Journal of Roentgenology: 1-6. 10.2214/ 
However, throat swab and PCR or genetic sequancing are the reliable confirmatory tests [1].

As ACE2 is mainly expressed in lungs, kidneys, gastrointestinal tract (GIT), and blood vessels, this might explain some of the COVID-19 induced clinical manifestaions, such as thrombo-embolism, dementia symptoms, renal and GIT viral localizations, thus the expected modes of transmission. Such manifestations are to be well considered within the therapeutic strategy, for example, the application of adequate anticoagulation [2].

https://mohammedshehatta1.wixsite.com/website/post/ sars-cov-2-covid-19

\section{Translational pathophysiology}

- SARS-CoV-2 infects its target cells through the attachment to cell surface receptor enzyme molecules, ACE2.

- ACE2 - Ang1-7 and ACE2 - Ang1-9 are 2 axises that play various roles in tissue protection (Figure 2).

- The virus attachment to the receptor blocks its protective roles, aggrevating tissue damage.

- Some important drugs that are widely used in our current medical practice, such as ibuprofen and angiotensin receptor blockers (ARBs), upregulates ACE2 expression, which might favor COVID-19 infection!! [3,4]. However, increased ACE2 expression might favor its protective functions, attenuating the disease manifestations!!

\section{Possible strategies}

Prevention is the best strategy

- Proper hygien

- Social distancing

- Frequent hand washing

- $\quad$ Staying home

Blocking viral replication

- There are some durgs that can block the RNA viral replication.

- Those drugs are safe \& approved for treatment of other viral infections, such as the relative corona viruses, MERS and or HIV.

- Clinical trials are conducted to test the efficacy of those drugs against SARS-CoV-2.

- Remdesivir \& Ciclesonide are examples

- Remdesivir showed the most satisfactory results and is either approved or under approval as the sole reliable anti-COVID-19 therapy, where its application to severely ill COVID-19 patients resulted in clinical improvement in 36 of 53 patients (68\%) [5].

Support against respiratory failure

As the COVID-19 infection results in respiratory failure,

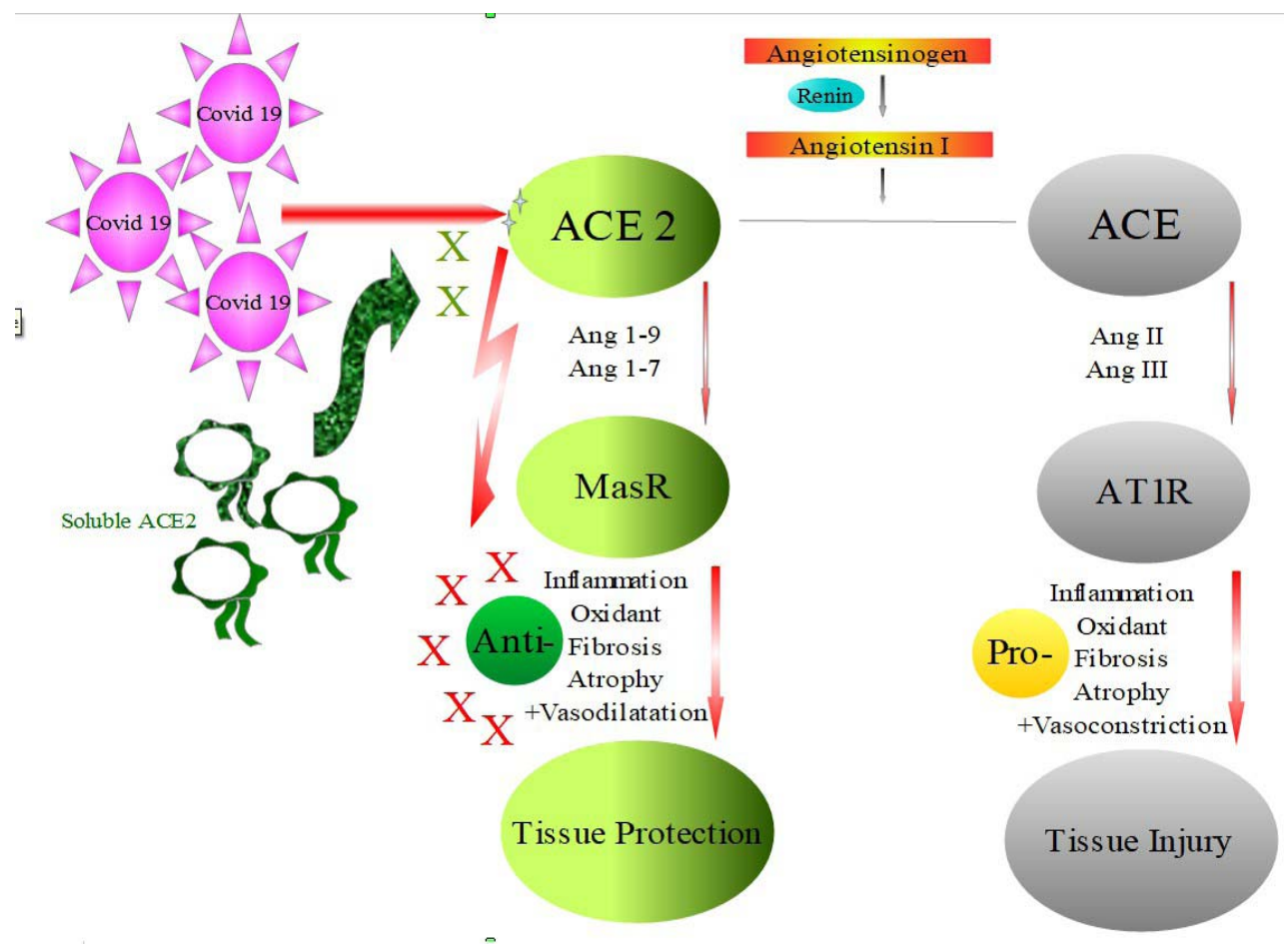


respiratory support is essential for patient's survival. In the intensive care, the critically ill patients are supported with mechanical ventilation. The aim is to maintain the necessary oxygenation, where CPAP machines could help in case of ventilators shortage.

Immunity Modulation

(1) Active immunization

- Thanks to the scientific efforts, the antigenic epitopes of the SARS-CoV-2 have been identified, where the genomic similarities between SARS-CoV-2 and SARS$\mathrm{CoV}$ reach up to $76.5 \%$. Thus, mRNA vaccine is under development and clinical validation.

- A coronavirus vaccine tested on humans has yielded promising early clinical data from the phase 1 study led by the National Institute of Allergy and Infectious Diseases (NIAID), the biotech company Moderna announced in a news release.

- Although didn't publish detailed data in a preprint or a scientific journal, the company said in its press release that all 45 participants across 3 study arms receiving different doses - 25, 100 or 250 micrograms of the vaccine - seroconverted 2 weeks after their first dose.

- Antibodies from 8 participants, who received doses of 25 and 100 micrograms, were tested for their ability to neutralize live SARS-CoV-2 in vitro, and all the 8 participants had neutralizing antibodies in amounts equal to or greater than those found in recovered patients.

(2) Passive immunization

- Not all infected persons die or even develop critical illness.

- Age, comorbidities, lung health, smoking, the amplitude of the immune reaction, and other factors, may determin the clinical outcome.

- Recovered patients develop antibodies and immunity against the SARS-CoV-2, which could be transferred to critically ill patients.

\section{(3) Cytokine storm modulation}

As previously explained, the body reaction to the virus might be worse than the infection itself, eliciting an immune reaction and releasing huge amounts of inflammatory cytokines that circulates freely in the body and lead to multiorgan failure, especially respiratory and renal failures. Thus, control of such an immune reaction is a therapeutic target, as important as the control of viral replication. CD24Fc

- Is a recombinant fusion protein composed of the extracellular domain of the mature human glycoprotein
CD24 linked to a human immunoglobulin G1 (IgG1) Fc domain, with potential immune checkpoint inhibitory, anti-inflammatory and antineoplastic activities.

- Upon administration, the CD24 extracellular domain-IgG1 Fc domain recombinant fusion protein CD24Fc binds to injured cell components, also called DAMPs (DangerAssociated Molecular Patterns), thereby preventing the interaction of DAMPs with toll-like receptors (TLRs) and inhibiting both nuclear factor-kappa B (NFkB) activation and secretion of inflammatory cytokines.

- In addition, CD24Fc binds to and activates Siglec G/10, a sialic acid-binding immunoglobulintype lectin, and stimulates SHP-1-mediated inhibitory signaling, preventing NFkB activation and secretion of inflammatory mediators, which further prevents inflammatory responses.

- Phase I clinical trial demonstrated its safety and ability to attenuate the expression of multiple inflammatory cytokines.

- Phase II clinical trial in patients of hematopoietic stem cell transplantation demonstrated its ability to attenuate severe (Grade 3-4) acute graft vs. host disease (GVHD), in which the transplanted T cells attack the recipient target tissues.

- Currently Phase II/III trials are being conducted for potential COVID-19 application [6].

Mesenchymal Stem Cells

- Mesenchymal stem cells (MSCs) are multipotent stromal cells that could be derived from adult bone marrow, as well as other tissues. These cells have the ability to differentiate into many cell lines, which gives a great hope that MSCs could be a magic regenerative therapy to restore damaged tissue.

- Mesenchymal stem cells are very tolerated by the immune system, where they can attenuate the immunity through modulating $\mathrm{T}$ cell activation and proliferation, either by a direct cell-cell interaction or via soluble factors.

- In addition, MSCs secrete many immunomodulatory factors that include IL10, transforming growth factor $\beta$ (TGF $\beta$ ), prostaglandin E2 (PGE2) and vascular endothelial growth factor (VEGF).

- IL10 is one of the major anti-inflammatory cytokine that down regulates the expression of cytokines in the $\mathrm{T}$ helper-1 cells, as well as the major histocompatibility class II antigens and costimulatory molecules on the surface of macrophages. Moreover, IL10 antagonizes the activity of NF- $\mathrm{KB}$ and is capable of suppressing the production of IL8 in a dose dependent manner. 
- While the endothelial adhesiveness increases significantly upon IL1 $\beta, T N F \alpha$, IFN $\gamma$, and Lipopolysaccharide stimulation, which increases the leukocytic (macrophages and lymphocytes) migration, TGF $\beta$, secreted by MSCs, has the ability to block these effects in a dose dependent manner.

- PGE2 functions towards theinhibition of the proliferation, differentiation and function of the antigen presenting, as well as the cytotoxic cells, including the dendritic cells, macrophages and natural killer (NK) cells.

- Thus MSCs have proved ability to modulate the adverse immune response and inflammation, as well as clinical safety in early phases of clinical trials.

- Clinical trials are being conducted to prove their benefits in COVID-19-19 cases [7].

(4) Hydroxychloroquine

- Is a drug used to treat malaria, lupus erythematosus and rheumatoid arthritis.

- It can attenuate inflammation through interfering with the antigen processing \& presentation, which is necessary to stimulate $\mathrm{CD} 4+\mathrm{T}$ cells , thus, it can be used as an immunity modulator to protect against the cytokine storm [8].

- Moreover, chloroquine analogs are able to inhibit the endosomal acidification and show an invitro antiviral activity at high micromolar concentrations against a broad range of emerging viruses, including COVID-19 [9].

- Clinical trials are being conducted for potential covid-19 application. However, the initial results show no evidence of a favourable clinical outcome. In other words, the in-vitro reported antiviral activities are still not reproducible clinically. In addition, the drug itself might have serious side effects $[10,11]$.

(5) ACE2 related strategies

- SARS-CoV-2 infects its target cells through attaching to the cell surface ACE2.

- ACE2 contributes to the attenuation of cytokine release [12].

- Treatment using rh-ACE2 may exert dual functions:

$>$ Slow down the viral entry into target cells and hence the viral spread

$>$ Protect the lung from injury (Figure 2) [13].

GSK2586881 is a rh-ACE2 that has been studied in Phase I trials few years ago for potential lung protection. Further investigations in Phase II trials confirmed its safety, the ability of significantly increase the levels of Ang1-7 and Ang1-5, as well as a tendency to decrease IL6 levels in plasma [14].

\section{MSAM recommendations}

Based on our translational immunology viewpoint, we think that SARS-CoV-2 can differ than influenza in the fact that:

- Both types of viruses infect pulmonary cells causing cells to die.

- Both infections trigger inflammatory responses up to cytokine storm.

- Influenza viruses interact with sialic acid residues of target cells surface glycans, keeping the protective roles of ACE2 intact.

- SARS-CoV-2 interacts with ACE2 of the target cells, abolishing its important protective signaling.

Accordingly, we highly recommend the therapeutic strategies to include drug combinations that can target the pathogenesis at many levels. For example, a combination of an effective anti-viral Remdesivir, soulable ACE2, and an immune modulator.

Alined with our recommendation, the early data from a small trial conducted by the University of Hong Kong has demonstrated promise for the COVID-19 treatment with a combination of HIV medicine lopinavir- ritonavir, hepatitis drug ribavirin, and multiple sclerosis therapy interferon beta. Such a combination demonstrates a strong anti-viral approach, combined with an immune modulator IF- $\beta$, and resulted in significant relive of symptoms, and reduction of the viral shedding duration and hospital stay in 86 patients with mild to moderate COVID-19, compared to 41 control patients [15].

\section{Conflicts of interest}

MSAM for medical consultations provide medical advisory services. This work is part of our contribution to the battle against the COVID-19 pandemy. There is no funding or commercial relations between MSAM for medical consultations and any of the products mentioned in the article.

\section{References}

1. Zhao W, Zhong Z, Xie X, Yu Q, Liu J, et al. Relation Between Chest CT Findings and Clinical Conditions of Coronavirus Disease (COVID-19) Pneumonia: A Multicenter Study. Am J Roentgenol. 2020; 214: 1072-1077. PubMed: https://www.ncbi.nlm.nih.gov/pubmed/32125873

2. Atallah B, Mallah SI, Al-Mahmeed W. Anticoagulation in COVID-19. Eur Heart J Cardiovasc Pharmacother. pvaa 036.

PubMed: https://www.ncbi.nlm.nih.gov/pubmed/32352517

3. Wanga $X$, Yea $Y$, Gong $H$, et al. The effects of different angiotensin II type 1 receptor blockers on the regulation of the ACE-Angll-AT1 and ACE2-Ang(1-7)-Mas axes in pressure overloadinduced cardiac remodeling in male mice. J Molecular Cellu Cardiol. 2016; 97: 180-190. 
4. Esler M, Esler D. Can Angiotensin Receptor-Blocking Drugs Perhaps Be Harmful in the COVID-19 Pandemic? J Hypertens. 2020; 38: 781-782. PubMed: https://pubmed.ncbi.nlm.nih.gov/32195824

5. Grein J, Ohmagari N, Shin D, et al. Compassionate Use of Remdesivir for Patients with Severe Covid-19. N Engl J Med. 2020; 2007016. PubMed: https://pubmed.ncbi.nlm.nih.gov/32275812

6. ClinicalTrials.gov Identifier: NCT04317040

7. ClinicalTrials.gov Identifier: NCT04313322

8. Fox RI. Mechanism of Action of Hydroxychloroquine as an Antirheumatic Drug. Semin Arthritis Rheum. 1993; 23(2 Suppl 1): 82-91.

PubMed: https://pubmed.ncbi.nlm.nih.gov/8278823/

9. Liu J, Cao R, Xu M, Wang $X$, et al. Hydroxychloroquine, a less toxic derivative of chloroquine, is effective in inhibiting SARS-CoV-2 infection in vitro. Cell Discovery. 2020; 6: 16.

PubMed: https://pubmed.ncbi.nlm.nih.gov/32194981/

10. ClinicalTrials.gov Identifier: NCT04307693

11. Molina JM, Delaugerre C, Le Goff J, Mela-Lima B, Ponscarme D, et al. No Evidence of Rapid Antiviral Clearance or Clinical Benefit With the
Combination of Hydroxychloroquine and Azithromycin in Patients With Severe COVID-19 Infection. Med Mal Infect. 2020; 50: 384.

PubMed: https://www.ncbi.nlm.nih.gov/pmc/articles/PMC7195369/

12. Prestes TRR, Rocha NP, Miranda AS, et al. The Antilnflammatory Potential of ACE2/Angiotensin-(1-7)/Mas Receptor Axis: Evidence from Basic and Clinical Research. Curr Drug Targets. 2017; 18: 1301-1313. PubMed: https://pubmed.ncbi.nlm.nih.gov/27469342/

13. Zhang H, Penninger JM, Li Y, Zhong N, Slutsky AS. Angiotensinconverting Enzyme 2 (ACE2) as a SARS-CoV-2 Receptor: Molecular Mechanisms and Potential Therapeutic Target. Intensive Care Med. 2020; 46: 586-590.

PubMed: https://pubmed.ncbi.nlm.nih.gov/32125455/

14. Khan A, Benthin C, Zeno B, Albertson TE, Boyd J, et al. A Pilot Clinical Trial of Recombinant Human Angiotensin-Converting Enzyme 2 in Acute Respiratory Distress Syndrome. Crit Care. 2017; 21: 234. PubMed: https://pubmed.ncbi.nlm.nih.gov/28877748

15. Hung IFN, Lung KC, Tso EV, Liu R, Chung TW, et al. Triple combination of interferon beta- $1 \mathrm{~b}$, lopinavir-ritonavir, and ribavirin in the treatment of patients admitted to hospital with COVID-19: an openlabel, randomised, phase 2 trial. The Lancet. 2020; 395: 1695-1704. PubMed: https://pubmed.ncbi.nlm.nih.gov/32401715/ 